\title{
Analytical Resource Optimization for the DF Relay-assisted Transmission under HARQ
}

\author{
Adrian Agustin, Josep Vidal and Olga Muñoz \\ Dept. Signal Theory and Communications, \\ Technical University of Catalonia (UPC), Barcelona, Spain
}

\begin{abstract}
Under limited channel state information at the transmitter the communication suffers from the outage events produced by a data rate selection not supported by the current channel realization. Automatic repeat request (ARQ) protocols are a useful tool to deal with those outage events in delay-tolerant services. This work looks into how Automatic Repeat Request (ARQ) protocols are applied to the half-duplex relay-assisted transmission with a decode-and-forward (DF) relay. The exploitation of ACK feedback channels from destination and relay leads to two possible implementations: 1) only-source manages the retransmission procedure or 2 ) both source and relay control the retransmissions. We analyze both options under a chase-combining ARQ protocol with limited number of transmission rounds, providing integral expressions for the throughput. This study allows the optimization transmission rate and the amount of resources allocated for the relay transmission.
\end{abstract}

Keywords-Hybrid ARQ, relay-based systems

\section{INTRODUCTION}

Relay-assisted transmission has shown its ability to combat the effects of propagation channel by introducing the relay terminal. Originally proposed in [1] with some capacity results proved in [2], it has regained attention due to the diversity gain obtained in wireless channels, [3],[4],[5],[6]. In this work the relay-assisted transmission assumes half-duplex relays under decode-and-forward (DF), [4], because current terminals cannot cope with simultaneous transmission and reception in the same band. Therefore, the relay-assisted transmission is divided in two orthogonal phases: relay-receive phase (or phase I) and relay-transmit phase (or phase II).

Among the many existing relaying protocols (see for instance [7]), in this work the relay-assisted transmission of a message is carried out in the following way, see Fig.1. In phase I the source transmits to the relay and destination. Afterwards, only the relay transmits to the destination. We do not consider the simultaneous transmission from source and relay. This is the same procedure used in [4] and named protocol I in [7]. The relay transmits using a different codebook from the source (space-time-coded cooperative diversity, [5]).

Multiple ACK feedback channels allow acknowledging the successful decoding of the messages to the source and/or relay. The exploitation of this knowledge and which terminal controls the retransmission procedure allows implementing the relayassisted transmission in different ways. For example, the source may manage the ARQ procedure [11] using the requests from the destination when simple relays are devised. If the

This work was supported in part by the European Union through project ROCKET ICT-2007-1-215282 and FEDER funds, and by the Spanish/Catalan Science and Technology Commissions through projects: 2005SGR-00639, TEC2006-06481/TCM, TEC2004-04526 and CONSOLIDER CSD200800010 COMONSENS destination cannot decode successfully the message, then the whole transmission is repeated (phase I and II). An ARQ protocol is proposed in [9], where the ACKs from relay and destination are required. It employs the relay only if it is able to decode the signal from the source in the first received phase I. Otherwise the source without the help of the relay deals with the subsequent retransmissions. The analysis is carried out for the pure $A R Q$ protocol [11] with adaptive modulation. This approach fails short of throughput when the source-destination link is of low quality. On the other hand, when both source and relay participate in the retransmissions, the source must be able to receive the feedback from the relay and destination while the relay listens the feedback from the destination. In such a case, the source transmits till either the relay or the destination decodes the message. Once the relay has decoded, the source is freed and only the relay retransmits to the destination. When applied to the downlink of cellular systems the resources are employed more efficiently, since the source-to-destination link is supposed to exhibit lower signal to noise ratio (SNR) than the relay-to-destination link. This ARQ protocol introduced in [10] follows the transmission sketched in Fig.1. That work outlines the benefits of using several assisting relays in each retransmission by means of a numerical evaluation of the throughput.

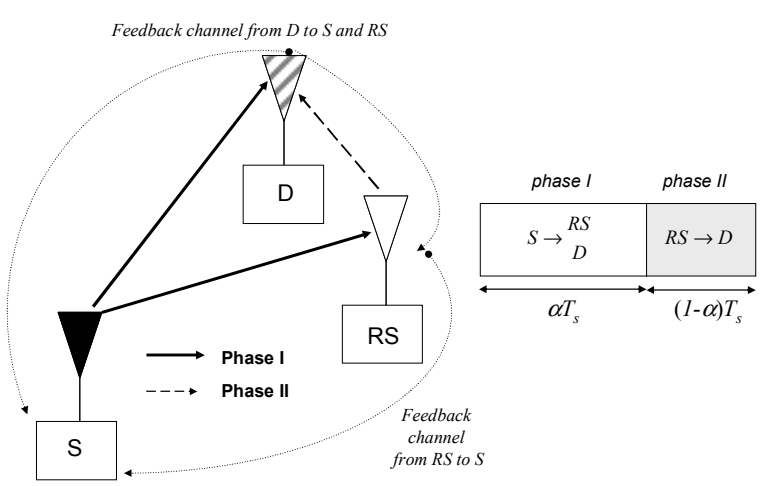

Figure 1. Relay-assisted transmission. S, RS and D denote source, relay and destination, respectively

The present work envisions the Hybrid ARQ type I (chase combining) [11] when only-source or source-and-relay manage the retransmission procedure. Unlike [9] and [10], the transmission rate and time of phases I and II are optimized to maximize the throughput. The main contributions are:

- We prove that the best throughput is obtained when source and relay manage the retransmission procedure. 
- We derive integral expressions for the throughput with a limited maximum number of transmissions.

- We elucidate which positions for the relay terminal are best to achieve the highest throughput.

\section{SCENARIO AND Signal MODEL}

Our scenario consists of a source, a half-duplex DF relay and a destination, all equipped with a single antenna. Source and relay transmit with constant power $P$ and Gaussian codewords. The transmission time is defined as time slot of duration $T_{s}$. For sake of notation simplicity the bandwidth allocated is $1 \mathrm{~Hz}$. We assume that the channel coefficients present an i.i.d. process with a Rayleigh distribution across successive retransmissions. The mutual information at the $i$-th link after $m$ transmissions under chase combining becomes,

$$
I_{i, m}=T_{s} \log _{2}\left(1+\sum_{j=1}^{m} \gamma_{i, j}\right) \text { bits }
$$

where $T_{\mathrm{s}}$ is the time slot duration and $\gamma_{i, j}$ is the instantaneous SNR of the $i$-th link in the $j$-th transmission round which follows an exponential distribution with mean,

$$
\rho_{i}=\frac{P}{L_{i} \sigma^{2}}
$$

where $i=0,1$ and 2 stand for source-destination, source-relay and relay-destination links, $L_{\mathrm{i}}$ defines the pathloss and $\sigma^{2}$ is the noise power at the destination and the relay.

The relay-assisted transmission is divided in two orthogonal phases of time duration $\alpha T_{s}$ and $(1-\alpha) T_{s}$ respectively (see Fig.1). The relay operates in DF and employs a different Gaussian codebook from the source, [5]. We characterize the success of the transmission by the outage probability, i.e. the probability that the selected data rate is not supported by the current channel realization. The performance of the transmission is measured in terms of throughput, so resources are optimized according to

$$
\mathcal{T}=\max _{B, \alpha} \frac{B}{E\{t\}}
$$

where $E\{t\}$ denotes the average time needed for successfully receiving $B$ bits. The feedback channels between the different terminals carry the one-bit ACK/NACK messages generated by the receivers at the destination and relay. If the relay manages the ARQ procedure, it must receive the feedback from destination. We assume that their transmissions are instantaneous and errorless. Likewise, the introduced overhead is beyond of the scope of the paper.

\section{A. Source manages the ARQ procedure}

Let us assume in this case that the source receives the ACK/NACK messages from the destination and relay, and it decides when a retransmission is required. Therefore, we identify two states (see Fig.2-left): $\mathrm{S}_{\mathrm{A}}$ where source and relay are transmitting to destination and $\mathrm{S}_{\mathrm{C}}$ where the destination has decoded the message. The transition between those states is evaluated after a complete relay-assisted transmission. Hence a transmission round for this scheme entails phase I and phase II.

The transmission is based on the protocol described by Fig.1 and follows the flowchart depicted in Fig.3-left. Notice that if the RS cannot decode the message, the source transmits again in phase II, leading to the selective $D F$ defined in [4], but here the source uses independent Gaussian codewords in both phases. Additionally, once the relay decodes the message, it does not evaluate any other possible retransmission from the source. Those transmissions will be used by the destination.

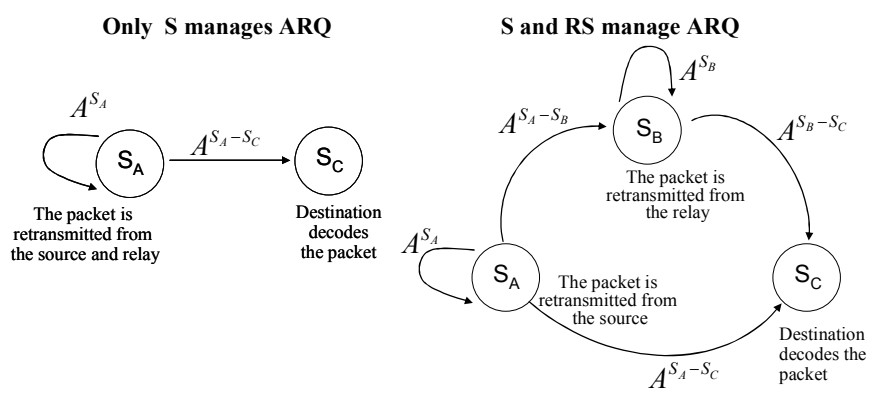

Figure 2. State diagram for the ARQ relay-assisted transmission. The ARQ procedure is managed by left) only-source and right) source and relay

Therefore, after $m$ transmission rounds and assuming that the RS was able to decode message in the $k$-th transmission, the mutual information obtained at the destination is given by,

$$
I_{30, k, m}=\alpha I_{0, m}+(1-\alpha) T_{s} \log _{2}\left(1+\sum_{j=1}^{k-1} \tilde{\gamma}_{0, j}+\sum_{j=k}^{m} \gamma_{2, j}\right)
$$

where $I_{0 . m}$ is defined by (1) and it stands for the combination of all the phases I, while the second term of (4) takes into account the phases II. In such a case, the source transmits during $k-1$ phases and the relay during $m-k$. The terms $\tilde{\gamma}_{0, j}, \gamma_{2, j}$ denote the instantaneous SNR of the source-destination and relaydestination links with Rayleigh distribution of mean (2). We have assumed that the instantaneous SNR of the sourcedestination link in phase I and phase II of the same transmission round is independent and identically distributed. Moreover, if the relay cannot decode the message during the $m$ transmission rounds, we will employ $I_{30, m+1, m}$, (4).

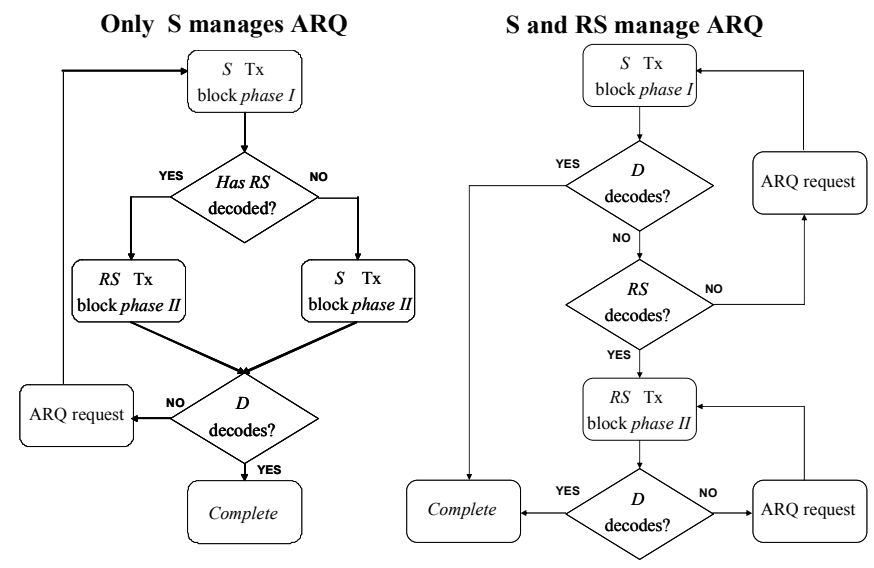

Figure 3. Flowchart of the relay-assisted transmission. (Left) only-source and (Right) source and relay manage the retransmission procedure

\section{B. Source and relay manage the ARQ procedure}

In this case source and relay are responsible of managing the retransmissions when a message is received wrongly at the destination. The source receives the ACK feedback from relay and destination, while the relay is also aware from the ACK/NACK messages from the destination. We have modeled 
this transmission by the three-state diagram sketched in Fig.2right. $S_{A}$ stands for the state where the source is transmitting to the relay and destination (phase I), controlling the ARQ procedure. $S_{B}$ defines the state where the relay has decoded the message and transmits to the destination (phase II), so only the relay is in charge of the ARQ procedure. Finally, $S_{C}$ represents that destination has decoded the message. The transmission follows the flowchart presented in Fig.3-right. The phase I will be repeated until the message is successfully decoded at the relay (or at the destination, in such a case a new message is transmitted by the source). Afterwards, relay transmissions in phase II are repeated until a successfully detection of the message at the destination. Notice that the destination is reporting an ACK/NACK message after each phase I in order to consider the transition from state $\mathrm{S}_{\mathrm{A}}$ to $\mathrm{S}_{\mathrm{C}}$. (Fig.2-right). It is important to stress that a transmission round consists of a single phase I or phase II, in contrast to the case of section II.A.

The mutual information at the destination obtained from combining $k$ and $m$ transmissions from the source and relay, respectively, is defined by,

$$
I_{20, k, m}=\alpha I_{0, k}+(1-\alpha) I_{2, m}
$$

\section{THROUGHPUT EVALUATION}

Our system accepts that some messages can be lost, modelled by a threshold on the packet drop probability $\left(P_{\mathrm{d}, \mathrm{thr}}\right)$. This event is due to the limited number of retransmissions $N_{L}$, so there is a non-zero probability of decoding the message, characterized by the packet drop probability $\left(P_{\mathrm{drop}}\right)$. In this work we evaluate the throughput by (3) imposing $P_{\text {drop }} \leq P_{\text {d,thr. }}$.

\section{A. Direct transmission}

The throughput evaluation of the direct transmission under Hybrid ARQ type I, see for example [12], can be derived from the state diagram presented in Fig.2-left using the following event definition,

$$
A_{m}^{0} \triangleq\left\{I_{0, m} \geq B\right\} \quad \bar{A}_{m}^{0} \triangleq\left\{I_{0, m}<B\right\}
$$

The transition probability between states will be defined by the probability of successfully decoding on the $m$-th transmission (and not before), defined as,

$$
q_{0}(m)=\operatorname{Pr}\left(\bar{A}_{1}^{0}, \ldots, \bar{A}_{m-1}^{0}\right)-\operatorname{Pr}\left(\bar{A}_{1}^{0}, \ldots, \bar{A}_{m-1}^{0}, \bar{A}_{m}^{0}\right)
$$

Since each time there is a retransmission the mutual information is increased, the probability of the sequence of mutual information at the destination $\left\{I_{0,1}, \ldots, I_{0, \mathrm{~m}}\right\}$ crosses level $B$ at the $m$-th step is not decreasing with probability 1 . It turns out that event $\bar{A}_{m}^{0}$ already includes all the previous events,

$$
\bar{A}_{l}^{0} \subseteq \bar{A}_{m}^{0} \quad \forall l<m
$$

Therefore, we can write (7) as,

$$
q_{0}(m)=\operatorname{Pr}\left(\bar{A}_{m-1}^{0}\right)-\operatorname{Pr}\left(\bar{A}_{m}^{0}\right)
$$

where the probability parameters are evaluated following (27) in the Appendix.

The packet drop probability is given by,

$$
P_{\text {drop }}=\operatorname{Pr}\left(\bar{A}_{1}^{0}, \ldots, \bar{A}_{N_{L}-1}^{0}, \bar{A}_{N_{L}}^{0}\right)=\operatorname{Pr}\left(\bar{A}_{N_{L}}^{0}\right)
$$

We also define the average transmitted time as,

$$
E\{t\}=\left\{\begin{array}{cl}
T_{s} \sum_{n=1}^{N_{L}} n q_{0}(n) & \text { if } P_{d r o p} \leq P_{d, t h r} \\
\infty & \text { otherwise }
\end{array}\right.
$$

Finally, the throughput is optimized using (3). Notice that if the packet drop probability is higher than the threshold (maximum allowed by the system) the total throughput becomes zero.

\section{B. Source manages the retransmission procedure}

Fig.2-left depicts the state diagram for evaluating the throughput when only-source manages the retransmission procedure. Similar to the previous case, when the destination is unable to decode the packet it requests a retransmission of the same packet. However, source and relay should consider the entire signal received in previous time instants. The throughput of the selective $D F$ is evaluated following the same guidelines as (6)-(11) but now using,

$$
\bar{A}_{m}^{S D F} \triangleq \bigcup_{k=1 \ldots m}\left(\left\{I_{30, k, m}<B\right\} \cap \Psi_{k}^{1}\right) \cup\left(\bar{A}_{m}^{1} \cap\left\{I_{30, m+1, m}<B\right\}\right)
$$

$$
A_{m}^{1}=\left\{\alpha I_{1, m} \geq B\right\}, \bar{A}_{m}^{1}=\left\{\alpha I_{1, m}<B\right\}, \Psi_{k}^{1}=\left\{\bar{A}_{1}^{1}, \ldots \bar{A}_{k-1}^{1}, A_{k}^{1}\right\}
$$

and $I_{30, \mathrm{k}, \mathrm{m}}$ defined in (4). The probability of $\Psi_{k}^{1}$ is obtained following similar steps as (7). The event $\Psi_{k}^{1}$ considers the case that the relay has decoded the message in the $k$-th transmission round, while $I_{30, \mathrm{~m}+1, \mathrm{~m}}$ considers the event that the relay has not decoded the message in none of the $m$ transmissions. The error events depend on the data rate $B$ and the resource allocation, $\alpha$. The probabilities of those events are calculated using (27) (for $\left.I_{l, m}\right)$ and (28) (for $\left.I_{30, k, m}\right)$.

\section{Source and relay manage the retransmission procedure}

The throughput in this case must consider the state diagram sketched in Fig.2-right. To this end, we define the transition probability after the $m$-th transmission as,

$$
\begin{aligned}
& A_{m}^{S_{A}} \triangleq \bar{A}_{m}^{0} \cap \bar{A}_{m}^{1}, \quad A_{m}^{S_{A}-S_{C}} \triangleq A_{m}^{0}, \quad A_{m}^{S_{A}-S_{B}} \triangleq \bar{A}_{m}^{0} \cap A_{m}^{1} \\
& A_{k, m}^{S_{B}} \triangleq\left\{I_{20, k, m}<B \mid \alpha I_{0, k}<B\right\}, \quad A_{k, m}^{S_{B}-S_{C}} \triangleq \bar{A}_{k, m}^{S_{B}}
\end{aligned}
$$

with

$$
A_{m}^{i} \triangleq\left\{\alpha I_{i, m} \geq B\right\} \quad \bar{A}_{m}^{i} \triangleq\left\{\alpha I_{i, m}<B\right\}
$$

If the relay does not participate in the transmission (transitions from state $S_{\mathrm{A}}$ to $S_{\mathrm{C}}$ ) we have the following probability of a successful decoding at the destination,

$$
\begin{aligned}
& q_{E_{0}}(m)=\operatorname{Pr}\left(A_{1}^{S_{A}}, \ldots, A_{m-1}^{S_{A}}, A_{m}^{S_{A}-S_{C}}\right)= \\
& =\operatorname{Pr}\left(\bar{A}_{1}^{1}, \ldots, \bar{A}_{m-1}^{1}\right)\left(\operatorname{Pr}\left(\bar{A}_{1}^{0}, \ldots, \bar{A}_{m-1}^{0}\right)-\operatorname{Pr}\left(\bar{A}_{1}^{0}, \ldots, \bar{A}_{m}^{0}\right)\right)= \\
& =\operatorname{Pr}\left(\bar{A}_{m-1}^{1}\right)\left(\operatorname{Pr}\left(\bar{A}_{m-1}^{0}\right)-\operatorname{Pr}\left(\bar{A}_{m}^{0}\right)\right) \quad 1 \leq m \leq N_{L}
\end{aligned}
$$

where we have considered equation (8). In such a case, the average transmission time is defined as,

$$
E\left\{t_{E_{0}}\right\}=T_{s} \sum_{n=1}^{N_{L}} n q_{E_{0}}(n)
$$

The packet drop probability under these circumstances is,

$$
P_{\text {drop }, E_{0}}=\operatorname{Pr}\left(\bar{A}_{N_{L}-1}^{1}\right) \operatorname{Pr}\left(\bar{A}_{N_{L}}^{0}\right)
$$


On the other hand, when the relay decodes successfully in the $k$-th transmission from the source, the destination is able to decode the transmission with probability $\left(S_{\mathrm{A}}-S_{\mathrm{B}}-S_{\mathrm{C}}\right.$ transitions),

$$
\begin{aligned}
& q_{E_{k}}(k, m)=\operatorname{Pr}\left(A_{1}^{S_{A}}, \ldots, A_{k-1}^{S_{A}}, A_{k}^{S_{A}-S_{B}}, A_{k, 1}^{S_{B}}, \ldots, A_{k, m}^{S_{B}-S_{C}}\right)= \\
& \quad=\operatorname{Pr}\left(\bar{A}_{k}^{0}\right)\left(\operatorname{Pr}\left(\bar{A}_{k-1}^{1}\right)-\operatorname{Pr}\left(\bar{A}_{k}^{1}\right)\right)\left(\operatorname{Pr}\left(\bar{A}_{k, m-1}^{S_{B}}\right)-\operatorname{Pr}\left(\bar{A}_{k, m}^{S_{B}}\right)\right)
\end{aligned}
$$

valid for all $1 \leq k \leq N_{L}, 1 \leq m<N_{L}-k$. Notice that the probabilities are evaluated using the equations derived in Appendix, (27) for events $\bar{A}_{k}^{0}, \bar{A}_{k}^{1}$ and (29) for event $\bar{A}_{k, j}^{S_{B}}$.

Now, the average transmission time becomes,

$$
E\left\{t_{E_{k}}\right\}=\sum_{n=1}^{N_{L}-k}\left(k \alpha T_{s}+n(1-\alpha) T_{s}\right) q_{E_{k}}(k, n), 1<k \leq N_{L}-1
$$

And the packet drop probability is defined as,

$$
P_{\text {drop }, E_{k}}=\operatorname{Pr}\left(\bar{A}_{k}^{0}\right)\left(\operatorname{Pr}\left(\bar{A}_{k-1}^{1}\right)-\operatorname{Pr}\left(\bar{A}_{k}^{1}\right)\right) \operatorname{Pr}\left(\bar{A}_{k, N_{L}-k}^{S_{B}}\right)
$$

The total packet drop probability of this system is defined as

$$
P_{d r o p}=\max _{0 \leq i \leq N_{L}-1} P_{d r o p, E_{i}}
$$

While we define the total average transmission time as,

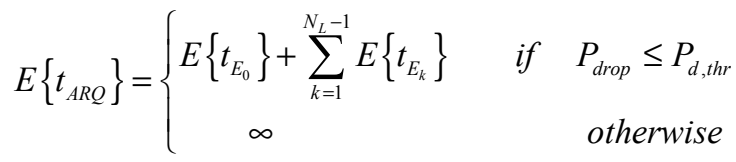

This definition produces zero-throughput solution, (3), when the $P_{\text {drop }}$ is higher than the threshold $\left(P_{\mathrm{d}, \text { thr }}\right)$

\section{RESULTS AND CONCLUSIONS}

The relay-assisted transmission is evaluated on a simple scenario where the source-destination distance is normalized to one. The RS is placed at distance $d$ from the source and (1- $d$ ) from the destination. The pathloss of each link $\left(L_{\mathrm{i}}\right)$ is proportional to the square of the distance between the terminals (propagation exponent equal to 2). The channel coefficients are Rayleigh distributed and the average SNR of each link is defined by (2). Notice that by defining the average SNR in the source-destination link, $\rho_{0}\left(L_{0}=1\right)$, we also define the average SNR of the other links, $\rho_{1}$ and $\rho_{2}$.

We will reference selective DF by S-DF-S (suffix-S due to the source manages the ARQ), while when source and relay manage the retransmission procedure we will use DF-SR. The results obtained in this section have assumed a maximum number of transmissions rounds $N_{L}$ and a threshold for the packet drop probability equal to $P_{\mathrm{d}, \mathrm{thr}}=10^{-3}$. Since the transmission rounds present a different resource allocation for both schemes, for a fair comparison we consider at maximum $N_{L} / 2$ phases I and $N_{L} / 2$ phases II for selective $D F$, while the sum of phases I and II is $N_{L}$ when source and relay manage the retransmission. In both cases the maximum number of times that source processes feedback messages is $N_{L}$.

Fig.4 presents the throughput attained by the different transmission schemes for $N_{L}=\{4,8\}$ and $\rho_{0}=0 \mathrm{~dB}$ as a function of the position of the relay. For a low value of $N_{L}$ the S-DF-S does not improve the direct transmission when RS is placed close to the source. However, the DF-SR gets significant throughput gains for all positions of the RS. By increasing $N_{L}$, the throughput is improved in all the schemes. We have observed (but not reported here) that the throughput is not significantly improved by using $N_{L}>8$ for this configuration.

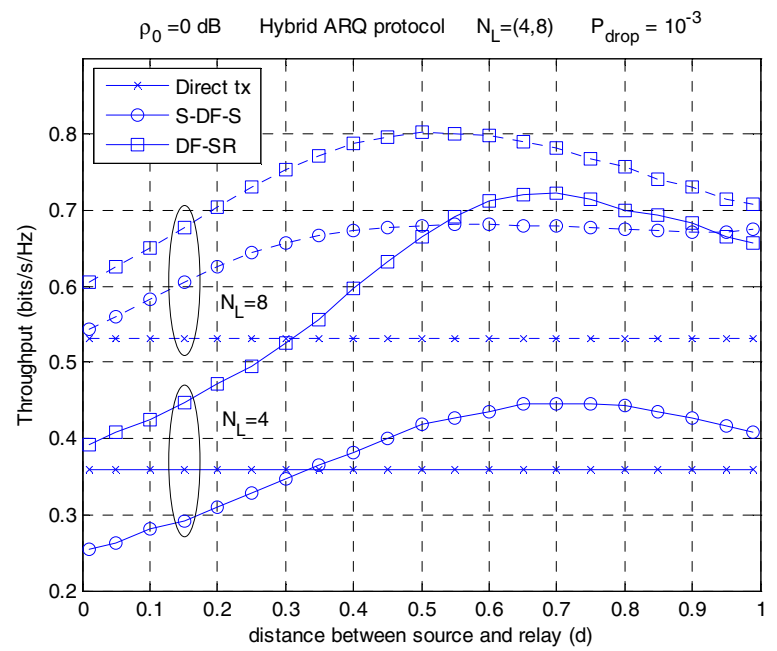

Figure 4. Throughput obtained by the different transmission strategies as a function of the distance between the source and the relay. $\rho_{0}=0$ dB. $N_{L}=\{4,8\}$

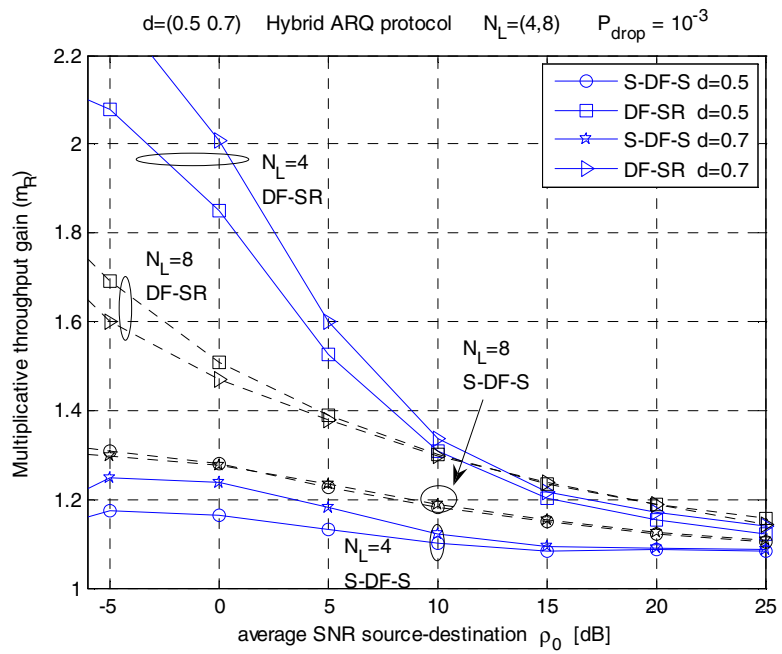

Figure 5. Multiplicative throughput gain $\left(m_{\mathrm{R}}\right)$ as a function of $\rho_{0}$. The RS is placed at $d=\{0.5,0.7\}$ from the source. $N_{\mathrm{L}}=\{4,8\}$

Fig.4 also showed that the positions of the relay where the maximum throughput is achieved depends on $N_{L}$. In this regard Fig.5 depicts the multiplicative throughput gain over the direct transmission $\left(m_{R}\right)$ when the $\mathrm{RS}$ is placed at $d=\{0.5,0.7\}$ for different values of $\rho_{0}$. Interestingly, for a large value of $N_{L}$, the position of the relay does not significantly influence the total gain, on the contrary of what happen for a low $N_{L}$. In all cases, the proposed protocol DF-SR outperforms $\mathrm{S}-\mathrm{DF}-\mathrm{S}$. The maximum throughput gains lie in the order of $25 \%$ at moderate SNR, while for low SNR get a $200 \%$ gain.

This work has provided integral expression for evaluating the througput of different relay schemes under HARQ type I, showing that the best scheme is that where source and relay control the retransmission procedure. Results have shown the ability of relay transmissions to provide homogeneous 
coverage in celullar systems. Additionally, the position of the RS becomes more relevant when the maximum number of retransmissions is small.

\section{APPENDIX}

This appendix unearths the expressions for the outage probability equations defined in previous sections when instantaneous SNR, $\gamma_{i}$, follows an exponential distribution with the mean $\rho_{i}$ defined in (2). In equations (1), (4) and (5) we have deal with the following random variables,

$$
x_{i, m}=\sum_{j=1}^{m} \gamma_{i, j} \quad w_{k, m}=\sum_{j=1}^{k-1} \tilde{\gamma}_{0, j}+\sum_{j=k}^{m} \gamma_{2, j}
$$

with probability density functions $(p d f)$ defined by,

$$
\begin{aligned}
& f_{x_{i, m}}(z)=\frac{z^{m-1}}{\Gamma(m) \rho_{i}^{m}} \exp \left(\frac{-z}{\rho_{i}}\right), \\
& f_{w_{k, m}}(z)=\frac{z^{m-1}{ }_{1} F_{1}\left(k, m+1, z\left(\rho_{2}^{-1}-\rho_{0}^{-1}\right)\right)}{\Gamma(m+1) \rho_{0}^{k} \rho_{2}^{m-k+1}} \exp \left(\frac{-z}{\rho_{2}}\right)
\end{aligned}
$$

where both equations are only defined for $z \geq 0$ and ${ }_{1} F_{1}()$ denotes the confluent hypergeometric function of the first kind, [14] If we are interested in the pdf of the $r=\beta \log _{2}(1+x)$ when the $p d f$ of the random variable $x$ is know (i.e. (24)), it can by obtained by

$$
f_{r}(z)=\frac{1}{\beta} \ln (2) 2^{\frac{z}{\beta}} f_{x}(\varphi(z / \beta)), \quad \varphi(y)=2^{y}-1
$$

For sake of notation simplicity in the equations to be presented we define,

$$
\lambda=\frac{B}{T_{s}}, \quad \bar{\alpha}=1-\alpha, \quad \omega\left(\rho_{i}, y, m\right)=1-\Gamma\left(m, \frac{\varphi(y)}{\rho_{i}}\right) \frac{1}{\Gamma(m)}(26)
$$

with function $\varphi$ defined in(25).

\section{A. Source-relay and relay-destination links}

The outage probability in the source-destination $(i=0)$ and source-relay $(i=1)$ links during phase $\mathrm{I}$ is,

$$
\operatorname{Pr}\left(\alpha I_{i, m}<B\right)=1-\Gamma\left(m, \frac{\varphi(\lambda / \alpha)}{\rho_{i}}\right) \frac{1}{\Gamma(m)}=\omega\left(\rho_{i}, \frac{\lambda}{\alpha}, m\right)
$$

\section{B. Source manages the $A R Q$}

The outage event at the destination after $m$ transmission rounds assuming the relay has decoded in the $k$-th retransmission, $\operatorname{Pr}\left(I_{30, k, m}<B\right)$ with $I_{30, \mathrm{k}, \mathrm{m}}$ defined in (4) is given by,

$$
\begin{aligned}
& \operatorname{Pr}\left(\alpha T_{s} \log _{2}\left(1+x_{0, m}\right)+(1-\alpha) T_{s} \log _{2}\left(1+w_{k, m}\right)<B\right)= \\
& =\operatorname{Pr}\left(\alpha I_{0, m}<\lambda-(1-\alpha) \psi\right)= \\
& =\int_{0}^{\lambda} \frac{1}{\beta} \ln (2) 2^{\frac{z}{\beta}} f_{w_{k, m}}(\varphi(z / \beta)) \omega\left(\rho_{0}, \frac{\lambda-z}{\alpha}, m\right) d z
\end{aligned}
$$

Notice that the $p d f$ of the random variables $I_{0, m}$ and $\psi=(1-\alpha) \log _{2}\left(1+w_{k, m}\right)$ is known thanks to $f_{r}$ defined in (25). The functions $\varphi$ and $\omega$ are introduced in (25) and (27). If the source is transmitting in both phases during all the transmission rounds, then we can use the same equation but using $k=m+1$. In the particular case of $k=1$, we should consider the variable $x_{k, m}$ (23) instead of $w_{k, m}$ in (28).

\section{Source and relay manage the $A R Q$}

Since the destination tries to decode the message after each phase, now the probability must be conditioned on the event that the destination was not able to successfully decode the message in phase I (state $S_{B}$ in Fig.3),

$$
\begin{aligned}
& \operatorname{Pr}\left(\alpha I_{0, k}+(1-\alpha) I_{2, m}<B \mid \alpha I_{0, k}<B\right)= \\
= & \int_{0}^{\lambda} \int_{0}^{\lambda-y} f_{\bar{\alpha} I_{2, m}}(y) f_{\alpha I_{0, k} \mid \alpha I_{0, k}<B}(x) d y d x= \\
= & \int_{0}^{\lambda} \frac{\ln (2) \varphi(y / \bar{\alpha})^{m-1} 2^{\frac{y}{\alpha}} \exp \left(\frac{-\varphi(y / \bar{\alpha})}{\rho_{2}}\right)}{\bar{\alpha} \Gamma(m) \rho_{2}^{m} \omega\left(\rho_{0}, \lambda / \alpha, k\right)} \omega\left(\rho_{0}, \frac{\lambda-y}{\alpha}, k\right) d y
\end{aligned}
$$

where we have assumed $f_{\alpha I_{0, k} \mid \alpha I_{0, k}<B}=f_{\alpha I_{0, k}} \omega\left(\rho_{0}, \lambda / \alpha, m\right)^{-1}$ and the $p d f$ of $\alpha I_{0, k}$ and $\omega$ are defined in (25)and (27).

\section{REFERENCES}

[1] E.C. van der Meulen, "Three-terminal communication channels", Adv. Appl. Prob., vol.3, pp. 120-154, 1971.

[2] T.M.Cover, A.El Gamal, "Capacity theorems for the relay channel", IEEE Trans. on Information Theory, vol.25,no.5,pp.1468-1489,Sep.1979

[3] A.Sendonaris, E.Erkip, B.Aazhang, "User cooperation diversity-part I: System description", IEEE Trans. on Communications, vol.51, no.11, pp. 1927-1938, Nov. 2003.

[4] J.N.Laneman, D.N.C.Tse, G.W.Wornell, "Cooperative diversity in wireless networks: Efficient protocols and outage behavior", IEEE Trans. Information Theory, vol. 50, no.12, pp.3062-3080, Dec. 2004.

[5] J.N.Laneman, G.W.Wornell, "Distributed Space-Time coded protocols for exploiting Cooperative Diversity in Wireless Networks", IEEE Trans. on Information Theory, vol. 49, no. 10, pp.2415-2425, Oct. 2003.

[6] G.Kramer, M.Gastpar, P.Gupta, "Cooperative strategies and capacity theorems for relay networks", IEEE Trans. on Information Theory, vol. 51, no. 9, Sep. 2005.

[7] A.Agustin, Relay-assisted transmission and Radio Resource Management for Wireless Networks, PhD Thesis, Technical University of Catalonia (UPC), May 2008, Barcelona, Spain.

[8] M.N. Khormuji, E.G.Larsson, "Analytical results on block length optimization for decode-and-forward relaying with CSI feedback", in Proc. IEEE Workshop on Signal Proc. Advances in Wireless Comms., June 2007, Helsinki, Finland.

[9] Z.Lin, E.Erkip, M.Ghosh, "Adaptive modulation for coded cooperative systems", in Proc. 6th IEEE Workshop on Signal Processing Advances in Wireless Communications (SPAWC), June 2005, New York, USA.

[10] B.Zhao, M.C.Valenti, "Practical Relay Networks: A Generalization of Hybrid-ARQ", IEEE Journal on Selected Areas in Communications, vol. 23, no.1, pp.7-18, Jan.2005.

[11] D.J.Costello, J.Hagenauer, H.Imai, S.B.Wicker, "Applications of errorcontrol coding”, IEEE Trans. Information Theory, vol.44, no.6, pp.25312560, Oct.1998.

[12] G.Caire, D.Tuninetti, "The Throughput of Hybrid-ARQ protocols for the Gaussian collision channel", IEEE Trans. on Inform. Theory, vol.47,no.5, pp.1971-1988, Jul.2001.

[13] M.K.Simon, Probability Distributions Involving Gaussian Random Variables: A handbook for engineers and Scientists. Springer 2002

[14] Abramowitz, M. and Stegun, I.A. (Eds.). "Confluent Hypergeometric Functions." Ch.13 in Handbook of Mathematical Functions with Formulas, Graphs, and Mathematical Tables, 9th printing. New York: Dover, pp.503-515, 1972. 